International Journal of Applied Mathematics

Volume 32 No. $3 \quad 2019,451-468$

ISSN: $1311-1728$ (printed version); ISSN: 1314-8060 (on-line version)

doi: http://dx.doi.org/10.12732/ijam.v32i3.7

\title{
ADOMIAN POLYNOMIAL AND ELZAKI TRANSFORM METHOD FOR SOLVING KLEIN GORDON EQUATIONS
}

\author{
Olufemi Elijah Ige ${ }^{1 \S}$, Razaq Adekola Oderinu ${ }^{2}$, Tarig M. Elzaki ${ }^{3}$ \\ ${ }^{1}$ Department of Mathematics and Physics \\ Lappeenranta University of Technology \\ P.O. Box 20, 53851 Lappeenranta, FINLAND \\ ${ }^{2}$ Department of Pure and Applied Mathematics \\ Ladoke Akintola University of Technology \\ P.M.B 4000, Oyo state, NIGERIA \\ ${ }^{3}$ Mathematics Department \\ Faculty of Science and Arts - Alkamil \\ University of Jeddah, Jeddah, SAUDI ARABIA
}

\begin{abstract}
In this paper, the combination of Elzaki transform and Adomian polynomial is used to obtain the approximate analytical solutions of nonlinear Klein Gordon equations. The approximate analytical solutions of all these equations are calculated in series form. In total, four Klein-Gordon equations from mathematical physics were considered to show the performance and effectiveness of this method. A three dimensional graph of solutions of some problems considered were plotted to show the shape of the solutions obtained and compared with that given in the references and they were found to agree. By comparing this method with some other known methods, all the problems considered showed that the Elzaki transform method and Adomian polynomial are very powerful and effective integral transform methods in solving some nonlinear equations.
\end{abstract}

AMS Subject Classification: 74J40, 74S30, 97N40

Key Words: Elzaki transform method, Adomian polynomial, Klein-Gordon equations

Received: January 25, 2019

(C) 2019 Academic Publications

${ }^{\S}$ Correspondence author 


\section{Introduction}

Klein-Gordon mathematical model is regarded as one of the most essential models in Quantum Mechanics. It is also applicable in collision plasma for interaction of solution as well as in condensed matter physics, initial state recurrence and nonlinear wave equation [22]. Moreover, this equation is very important in mathematical physics such as fluid dynamics, solid state physics and chemical kinetics [1], [5], [9].

Generally, the Klein-Gordon equation has the form:

$$
u_{t t}-u_{x x}+N u(x, t)=a(x, t),
$$

with the initial conditions

$$
u(x, 0)=b(x), \quad u_{t}(x, 0)=c(x),
$$

where $u$ is a function of $x$ and $t, N u(x, t)$ denotes nonlinear function and $a(x, t)$ is a known analytic function, [19].

Several methods have been developed to obtain the approximate analytical solutions of Klein-Gordon equations and some nonlinear differential equations, some of these methods are Exp function method [10], Reduced differential transform method, the Homotopy analysis method [23], Adomian decomposition method [2], [11], [21], [25], Variation iteration method [3], [4], [24], [26] and Homotopy perturbation method [20].

In this paper, we find the solutions of nonlinear Klein-Gordon equations by Elzaki transform method (ETM) and Adomian Polynomial. This method gives the solutions as an approximate analytical solutions in series form and most of time, it yield exact solutions with few iterations.

The structure of this paper is organized as follows: Section 2 contains the basic definitions and the properties of the proposed method. Section 3 shows the theoretical approach of the proposed method on Klein-Gordon equations. In Section 4, we apply the Elzaki transform method and Adomian polynomial to solve four problems in order to show its efficiency.

\section{Properties of Elzaki transform}

The Elzaki transform [7], [8], [11], [12], [13], [14], [15], [16], [17] is defined for functions of exponential order [13]. Consider the functions in the set $A$ define below

$$
A=\left\{f(t): \exists M, c_{1}, c_{2}>0,|f(t)|<M e^{\frac{|t|}{c_{j}}}, \text { if } t \in(-1)^{j} \times[0, \infty)\right\} .
$$


For any given function in the set $A$ defined above, the constants $c_{1}, c_{2}$ may be either finite or infinite, but $M$ must be infinite.

According to Tarig [13], the Elzaki transform is defined as:

$$
E[f(t)]=u^{2} \int_{0}^{\infty} f(u t) e^{-t} d t=T(u), \quad t \geq 0, u \in\left(c_{1}, c_{2}\right),
$$

or

$$
E[f(t)]=u \int_{0}^{\infty} f(t) e^{-\frac{t}{u}} d t=T(u), \quad t \geq 0, u \in\left(c_{1}, c_{2}\right)
$$

where $u$ is used to factor $t$ in the analysis of function $f$.

Let $T(u)$ be the Elzaki transform of $f(t)$ such that $E[f(t)]=T(u)$. Then,

(i) $E\left[f^{\prime}(t)\right]=\frac{T(u)}{u}-u f(0)$,

(ii) $E\left[f^{\prime \prime}(t)\right]=\frac{T(u)}{u^{2}}-f(0)-u f^{\prime}(0)$,

(iii) $E\left[f^{(n)}(t)\right]=\frac{T(u)}{u^{n}}-\sum_{k=0}^{n-1} u^{2-n+k} f^{(k)}(0)$.

$E[f(t)]=T(u)$ means that $T(u)$ is the Elzaki transform of $f(t)$, and $f(t)$ is the inverse Elzaki transform of $T(u)$. That is,

$$
f(t)=E^{-1}[T(u)] .
$$

In order to obtain the Elzaki transform of a partial derivative, integration by part is used on the definition of Elzaki transform and the resulting expressions are (see [18]):

$$
\begin{aligned}
& E\left[\frac{\partial f(x, t)}{\partial t}\right]=\frac{T(x, v)}{v}-v f(x, 0), \\
& E\left[\frac{\partial^{2} f(x, t)}{\partial t^{2}}\right]=\frac{T(x, v)}{v^{2}}-f(x, 0)-v \frac{\partial f(x, 0)}{\partial t}, \\
& E\left[\frac{\partial f(x, t)}{\partial x}\right]=\frac{d}{d x}[T(x, v)], \\
& E\left[\frac{\partial^{2} f(x, t)}{\partial x^{2}}\right]=\frac{d^{2}}{d x^{2}}[T(x, v)] .
\end{aligned}
$$




\section{Theoretical Approach: Elzaki transform on the Klein-Gordon equation}

The focus of this paper is to solve the nonlinear partial differential equations which are Klein-Gordon equations, we considered how Adomian polynomial is integrated into the Elzaki transform method to obtain the approximate analytic solutions of the aforementioned equations.

According to [28], consider

$$
\frac{\partial^{w} u(x, t)}{\partial t^{w}}+R u(x, t)+N u(x, t)=f(x, t)
$$

where $w=1,2,3$, and the initial conditions is given as

$$
\left.\frac{\partial^{w-1} u(x, t)}{\partial t^{w-1}}\right|_{t=0}=g_{w-1}(x) .
$$

The partial derivative of the function $u(x, t)$ of $w^{t h}$ order is the one given as $\frac{\partial^{w} u(x, t)}{\partial t^{w}}, R$ represents the linear differential operator, $N$ indicates the nonlinear terms of differential equations, and $f(x, t)$ is the non-homogeneous/source term.

By applying the Elzaki transform on equation (4) we have:

$$
E\left[\frac{\partial^{w} u(x, t)}{\partial t^{w}}\right]+E[R u(x, t)]+E[N u(x, t)]=E[f(x, t)],
$$

where

$$
E\left[\frac{\partial^{w} u(x, t)}{\partial t^{w}}\right]=\frac{E[u(x, t)]}{v^{w}}-\sum_{k=0}^{w-1} v^{2-w+k} \frac{\partial^{k} u(x, 0)}{\partial t^{k}} .
$$

Substituting equation (6) into equation (5) gives:

$$
\begin{aligned}
& \frac{E[u(x, t)]}{v^{w}}-\sum_{k=0}^{w-1} v^{2-w+k} \frac{\partial^{k} u(x, 0)}{\partial t^{k}}+E[R u(x, t)]+E[N u(x, t)] \\
& =E[f(x, t)] .
\end{aligned}
$$

This can be written as

$$
\frac{E[u(x, t)]}{v^{w}}=E[f(x, t)]+\sum_{k=0}^{w-1} v^{2-w+k} \frac{\partial^{k} u(x, 0)}{\partial t^{k}}
$$




$$
-\{E[R u(x, t)]+E[N u(x, t)]\} .
$$

By simplifying equation (7) we get

$$
\begin{aligned}
E[u(x, t)]= & v^{w} E[f(x, t)]+\sum_{k=0}^{w-1} v^{2+k} \frac{\partial^{k} u(x, 0)}{\partial t^{k}} \\
& -v^{w}\{E[R u(x, t)]+E[N u(x, t)]\} .
\end{aligned}
$$

Applying the inverse Elzaki transform to equation (8)

$$
\begin{aligned}
u(x, t)= & E^{-1}\left[v^{w} E[f(x, t)]+\sum_{k=0}^{w-1} v^{2+k} \frac{\partial^{k} u(x, 0)}{\partial t^{k}}\right] \\
& -E^{-1}\left[v^{w}\{E[R u(x, t)]+E[N u(x, t)]\}\right] .
\end{aligned}
$$

We can rewrite this as

$$
u(x, t)=F(x, t)-E^{-1}\left[v^{w}\{E[R u(x, t)]+E[N u(x, t)]\}\right],
$$

where $F(x, t)$ denotes the expression that arises from the given initial condition and the source terms after simplification.

The solution will be in the form of infinite series as

$$
u(x, t)=\sum_{n=0}^{\infty} u_{n}(x, t) .
$$

We can also decompose the nonlinear term as

$$
N u(x, t)=\sum_{n=0}^{\infty} A_{n},
$$

where $A_{n}$ are defined as the Adomian polynomials which can be calculated by using the formula $([27])$

$$
A_{n}=\frac{1}{n !} \frac{\partial^{n}}{\partial \lambda^{n}}\left[N\left(\sum_{i=0}^{\infty} \lambda^{i} u_{i}\right)\right]_{\lambda=0}, \quad n=0,1, \cdots
$$

Substituting equation (10) and equation (11) into equation (9) yields

$$
\sum_{n=0}^{\infty} u_{n}(x, t)=
$$




$$
F(x, t)-E^{-1}\left[v^{w}\left\{E\left[R \sum_{n=0}^{\infty} u_{n}(x, t)\right]+E\left[\sum_{n=0}^{\infty} A_{n}\right]\right\}\right] .
$$

Then from equation (12),

$$
u_{0}(x, t)=F(x, t),
$$

and the recursive relation is given as;

$$
u_{n+1}=-E^{-1}\left[v^{w}\left\{E\left[R u_{n}(x, t)\right]+E\left[A_{n}\right]\right\}\right] .
$$

Here $w=1,2,3$ and $n \geq 0$. The analytical solution $u(x, t)$ can be approximated by a truncated series

$$
u(x, t)=\lim _{N \rightarrow \infty} \sum_{n=0}^{N} u_{n}(x, t) .
$$

\section{Applications}

The effectiveness of the Elzaki transform and Adomian polynomials is demonstrated by solving the following Klein-Gordon equations.

Example 4.1: Consider the inhomogeneous nonlinear Klein-Gordon Equation $[6]$

$$
u_{t t}-u_{x x}+u^{2}=6 x t\left(x^{2}-t^{2}\right)+x^{6} t^{6},
$$

with initial conditions

$$
u(x, 0)=0, \quad u_{t}(x, 0)=0 .
$$

Applying the Elzaki transform to both sides of equation (13), gives

$$
E\left[u_{t t}\right]-E\left[u_{x x}\right]=E\left[6 x t\left(x^{2}-t^{2}\right)+x^{6} t^{6}\right]-E\left[u^{2}\right],
$$

where

$$
\begin{aligned}
& E\left[u_{t t}\right]=\frac{U(x, v)}{v^{2}}-u(x, 0)-v u_{t}(x, 0), \\
& E\left[u_{x x}\right]=\frac{d^{2}}{d x^{2}}[U(x, v)]=\frac{d^{2}}{d x^{2}} E[u] .
\end{aligned}
$$


Equation (14) becomes

$$
\begin{aligned}
& \frac{U(x, v)}{v^{2}}-u(x, 0)-v u_{t}(x, 0)-\frac{d^{2}}{d x^{2}} E[u] \\
& =E\left[6 x t\left(x^{2}-t^{2}\right)+x^{6} t^{6}\right]-E\left[u^{2}\right] .
\end{aligned}
$$

Applying the given initial conditions to equation (15) and simplifying, we obtain

$$
U(x, v)=6 x^{3} v^{5}-36 x v^{7}+720 x^{6} v^{10}+v^{2} \frac{d^{2}}{d x^{2}} E[u]-v^{2} E\left[u^{2}\right] .
$$

Applying the inverse Elzaki transform to equation (16) and simplifying, we have

$$
u(x, t)=x^{3} t^{3}-\frac{3}{10} x t^{5}+\frac{1}{56} x^{6} t^{8}+E^{-1}\left\{v^{2} \frac{d^{2}}{d x^{2}} E[u]-v^{2} E\left[u^{2}\right]\right\} .
$$

From equation (17), let

$$
u_{0}=x^{3} t^{3}-\frac{3}{10} x t^{5}+\frac{1}{56} x^{6} t^{8} .
$$

Now the recursive relation is given as

$$
u_{n+1}=E^{-1}\left\{v^{2} \frac{d^{2}}{d x^{2}} E\left[u_{n}\right]-v^{2} E\left[A_{n}\right]\right\} .
$$

$A_{n}$ is the Adomian polynomial to decompose the nonlinear terms by using the relation

$$
A_{n}=\frac{1}{n !} \frac{d^{n}}{d \lambda^{n}} f\left[\sum_{i=0}^{\infty} \lambda^{i} u_{i}\right]_{\lambda=0}
$$

Let the nonlinear term be represented by

$$
f(u)=u^{2} .
$$

Using equation (21) in equation (20), we obtain

$$
A_{0}=u_{0}^{2}, \quad A_{1}=2 u_{0} u_{1}, \quad A_{2}=2 u_{0} u_{2}+u_{1}^{2}, \quad \cdots .
$$

From equation (19), when $n=0$, we have

$$
u_{1}=E^{-1}\left\{v^{2} \frac{d^{2}}{d x^{2}} E\left[u_{0}\right]-v^{2} E\left[A_{0}\right]\right\} .
$$


For simplicity let us use $u_{0}=x^{3} t^{3}$, then $A_{0}=u_{0}^{2}=x^{6} t^{6}$, which yields:

$$
u_{1}=E^{-1}\left\{v^{2} \frac{d^{2}}{d x^{2}} E\left[x^{3} t^{3}\right]-v^{2} E\left[x^{6} t^{6}\right]\right\} .
$$

By simplifying equation (22) we get

$$
u_{1}=\frac{3}{10} x t^{5}-\frac{1}{56} x^{6} t^{8} .
$$

The approximate series solution is

$$
u(x, t)=u_{0}+u_{1}+\cdots
$$

Therefore,

$$
u(x, t)=x^{3} t^{3}
$$

Figure 1 below shows the 3D graph of the solution of equation (13), [6].

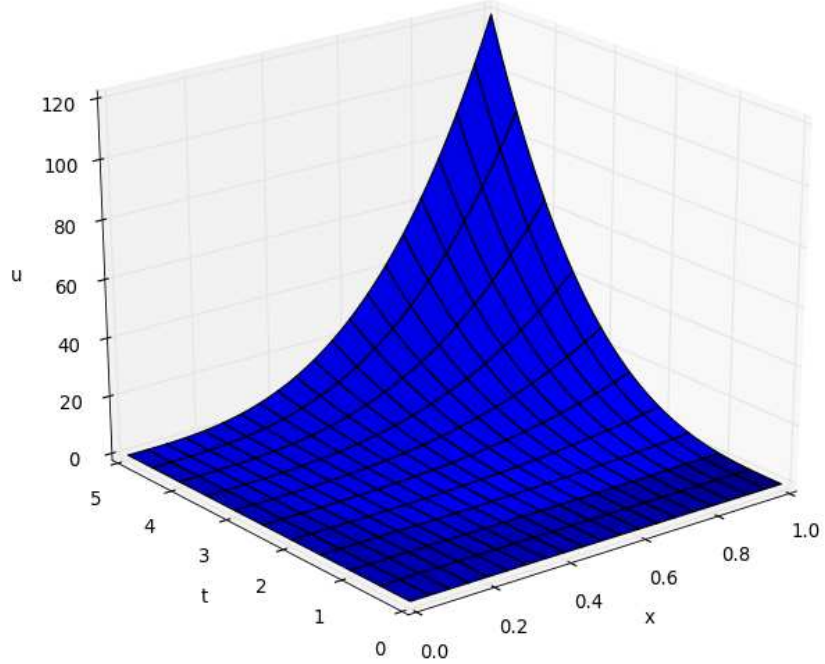

Figure 1: The solution of the first Klein-Gordon equation by ETM in equation (13)

Example 4.2: Consider the inhomogeneous nonlinear Klein-Gordon equation [6], [19]

$$
u_{t t}-u_{x x}+u^{2}=-x \cos t+x^{2} \cos ^{2} t,
$$


with initial conditions

$$
u(x, 0)=x, \quad u_{t}(x, 0)=0 .
$$

Applying the Elzaki transform to both sides of equation (25) gives

$$
E\left[u_{t t}\right]-E\left[u_{x x}\right]=E\left[-x \cos t+x^{2} \cos ^{2} t\right]-E\left[u^{2}\right]
$$

where

$$
\begin{aligned}
& E\left[u_{t t}\right]=\frac{U(x, v)}{v^{2}}-u(x, 0)-v u_{t}(x, 0), \\
& E\left[u_{x x}\right]=\frac{d^{2}}{d x^{2}}[U(x, v)]=\frac{d^{2}}{d x^{2}} E[u] .
\end{aligned}
$$

Equation (26) becomes

$$
\begin{aligned}
& \frac{U(x, v)}{v^{2}}-u(x, 0)-v u_{t}(x, 0)-\frac{d^{2}}{d x^{2}} E[u] \\
& =E\left[-x \cos t+x^{2} \cos ^{2} t\right]-E\left[u^{2}\right] .
\end{aligned}
$$

Applying the given initial conditions to equation (27) and simplifying, we obtain

$$
U(x, v)=x v^{2}+v^{2} E\left[-x \cos t+x^{2} \cos ^{2} t\right]+v^{2} \frac{d^{2}}{d x^{2}} E[u]-v^{2} E\left[u^{2}\right] .
$$

Applying the inverse Elzaki transform to equation (28) and simplifying yields

$$
u(x, t)=x \cos t+\frac{x^{2}}{2} t^{2}-\frac{x^{2}}{12} t^{4}+E^{-1}\left\{v^{2} \frac{d^{2}}{d x^{2}} E[u]-v^{2} E\left[u^{2}\right]\right\} .
$$

From equation (29), let

$$
u_{0}=x \cos t+\frac{x^{2}}{2} t^{2}-\frac{x^{2}}{12} t^{4}
$$

and the recursive relation is given as

$$
u_{n+1}=E^{-1}\left\{v^{2} \frac{d^{2}}{d x^{2}} E\left[u_{n}\right]-v^{2} E\left[A_{n}\right]\right\},
$$

where $A_{n}$ is the Adomian polynomial to decompose the nonlinear terms by using the relation

$$
A_{n}=\frac{1}{n !} \frac{d^{n}}{d \lambda^{n}} f\left[\sum_{i=0}^{\infty} \lambda^{i} u_{i}\right]_{\lambda=0}
$$


Let the nonlinear term be represented by

$$
f(u)=u^{2} .
$$

Substituting equation (33) in equation (32), we obtain

$$
A_{0}=u_{0}^{2}, \quad A_{1}=2 u_{0} u_{1}, \quad A_{2}=2 u_{0} u_{2}+u_{1}^{2}, \quad \cdots .
$$

From equation (31), when $\mathrm{n}=0$, we have

$$
u_{1}=E^{-1}\left\{v^{2} \frac{d^{2}}{d x^{2}} E\left[u_{0}\right]-v^{2} E\left[A_{0}\right]\right\} .
$$

For simplicity $u_{0}=x \cos t$ is used, then $A_{0}=u_{0}^{2}=x^{2} \cos ^{2} t$, and this yields

$$
u_{1}=E^{-1}\left\{v^{2} \frac{d^{2}}{d x^{2}} E[x \cos t]-v^{2} E\left[x^{2} \cos ^{2} t\right]\right\} .
$$

By simplifying equation (34) we obtain

$$
\begin{gathered}
u_{1}=-\frac{x^{2}}{2} E^{-1}\left[v^{4}+\frac{v^{4}}{1+4 v^{2}}\right] \\
u_{1}=-\frac{x^{2}}{2} t^{2}+\frac{x^{2}}{12} t^{4} .
\end{gathered}
$$

The approximate series solution is

$$
\begin{gathered}
u(x, t)=u_{0}+u_{1}+\cdots \\
u(x, t)=x \cos t+\frac{x^{2}}{2} t^{2}-\frac{x^{2}}{12} t^{4}-\frac{x^{2}}{2} t^{2}+\frac{x^{2}}{12} t^{4} .
\end{gathered}
$$

Therefore,

$$
u(x, t)=x \cos t
$$

Figure 2 below shows the 3D graph of the solution of equation (25), [6].

Example 4.3: Consider the nonlinear Klein-Gordon equation [19]

$$
u_{t t}-u_{x x}+\frac{3}{4} u-\frac{3}{2} u^{3}=0
$$




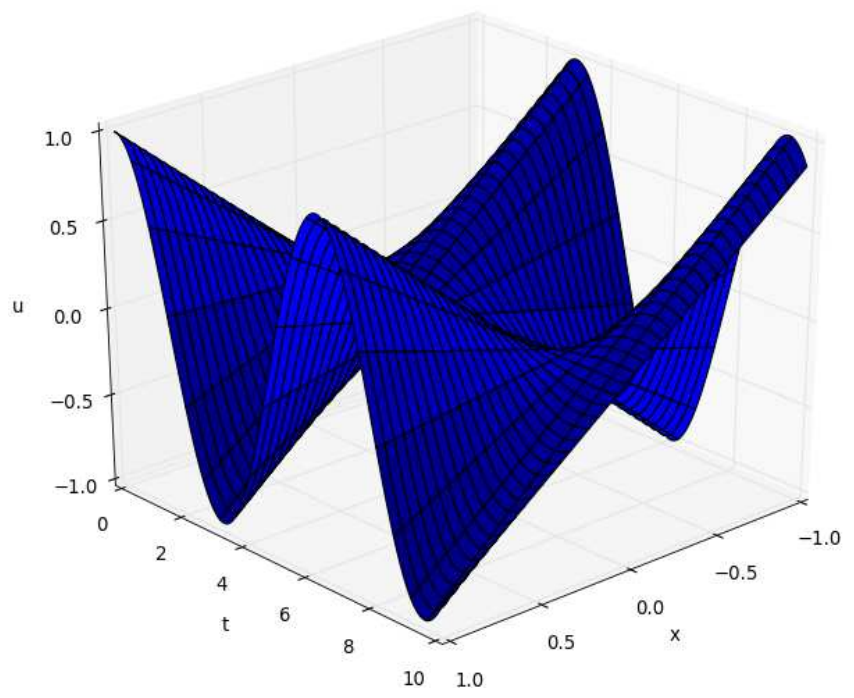

Figure 2: The solution of the second Klein-Gordon equation by ETM in equation (25)

with initial conditions

$$
u(x, 0)=-\operatorname{sech} x, \quad u_{t}(x, 0)=\frac{1}{2} \operatorname{sech} x \tanh x .
$$

Applying the Elzaki transform to both sides of equation (37), this gives

$$
E\left[u_{t t}\right]-E\left[u_{x x}\right]=-\frac{3}{4} E[u]+\frac{3}{2} E\left[u^{3}\right] .
$$

Recall that

$$
\begin{aligned}
& E\left[u_{t t}\right]=\frac{U(x, v)}{v^{2}}-u(x, 0)-v u_{t}(x, 0), \\
& E\left[u_{x x}\right]=\frac{d^{2}}{d x^{2}}[U(x, v)]=\frac{d^{2}}{d x^{2}} E[u] .
\end{aligned}
$$

Equation (38) becomes

$$
\frac{U(x, v)}{v^{2}}-u(x, 0)-v u_{t}(x, 0)-\frac{d^{2}}{d x^{2}} E[u]=-\frac{3}{4} E[u]+\frac{3}{2} E\left[u^{3}\right] .
$$

Applying the given initial conditions to equation (39) and simplifying, we obtain

$$
U(x, v)=-v^{2} \operatorname{sech} x+\frac{v^{3}}{2} \operatorname{sech} x \tanh x+v^{2} \frac{d^{2}}{d x^{2}} E[u]
$$




$$
-\frac{3 v^{2}}{4} E[u]+\frac{3 v^{2}}{2} E\left[u^{3}\right]
$$

Applying the inverse Elzaki transform to equation (40)

$$
\begin{aligned}
u(x, t) & =-\operatorname{sech} x+\frac{t}{2} \operatorname{sech} x \tanh x \\
& +E^{-1}\left\{v^{2} \frac{d^{2}}{d x^{2}} E[u]-\frac{3 v^{2}}{4} E[u]+\frac{3 v^{2}}{2} E\left[u^{3}\right]\right\}
\end{aligned}
$$

From equation (41), let

$$
u_{0}=-\operatorname{sech} x+\frac{t}{2} \operatorname{sech} x \tanh x
$$

We can express $u_{0}$ as

$$
u_{0}=-\frac{1}{\cosh x}+\frac{\sinh x}{2 \cosh ^{2} x} t
$$

and the recursive relation is given as

$$
u_{n+1}=E^{-1}\left\{v^{2} \frac{d^{2}}{d x^{2}} E\left[u_{n}\right]-\frac{3 v^{2}}{4} E\left[u_{n}\right]+\frac{3 v^{2}}{2} E\left[A_{n}\right]\right\},
$$

where $A_{n}$ is the Adomian polynomial to decompose the nonlinear terms by using the relation

$$
A_{n}=\frac{1}{n !} \frac{d^{n}}{d \lambda^{n}} f\left[\sum_{i=0}^{\infty} \lambda^{i} u_{i}\right]_{\lambda=0}
$$

Let the nonlinear term be represented by

$$
f(u)=u^{3}
$$

Substituting equation (44) into equation (43), we obtain

$$
A_{0}=u_{0}^{3}, \quad A_{1}=3 u_{1} u_{0}^{2}, \quad A_{2}=3 u_{0} u_{1}^{2}+3 u_{2} u_{0}^{2}, \quad \cdots .
$$

When $n=0$, we have

$$
u_{1}=E^{-1}\left\{v^{2} \frac{d^{2}}{d x^{2}} E\left[u_{0}\right]-\frac{3 v^{2}}{4} E\left[u_{0}\right]+\frac{3 v^{2}}{2} E\left[A_{0}\right] .\right\} .
$$




$$
u_{1}=E^{-1}\left\{v^{2} \frac{d^{2}}{d x^{2}} E\left[u_{0}\right]\right\}-E^{-1}\left\{\frac{3 v^{2}}{4} E\left[u_{0}\right]\right\}+E^{-1}\left\{\frac{3 v^{2}}{2} E\left[A_{0}\right]\right\} .
$$

$A_{0}$ is computed as:

$$
\begin{aligned}
A_{0}= & -\operatorname{sech}^{3} x+\frac{3}{2} t \operatorname{sech}^{3} x \tanh x \\
& -\frac{3}{4} t^{2} \operatorname{sech}^{3} x \tanh ^{2} x+\frac{1}{8} t^{3} \operatorname{sech}^{3} x \tanh ^{3} x .
\end{aligned}
$$

Therefore,

$$
u_{1}=-t^{2}\left[\frac{\cosh ^{2} x-2}{8 \cosh ^{3} x}\right]+t^{3} \frac{\left[\cosh ^{2} x-6\right] \sinh x}{48 \cosh ^{4} x}+\cdots
$$

The approximate series solution is

$$
\begin{gathered}
u(x, t)=u_{0}+u_{1}+\cdots \\
u(x, t)=-\frac{1}{\cosh x}+\frac{\sinh x}{2 \cosh ^{2} x} t-\frac{\left[\cosh ^{2} x-2\right]}{8 \cosh ^{3} x} t^{2} \\
+\frac{\left[\cosh ^{2} x-6\right] \sinh ^{3}}{48 \cosh ^{4} x} t^{3}+\cdots
\end{gathered}
$$

Figure 3 below shows the 3D graph of the solution of equation (37), [19].

Example 4.4: Consider the homogeneous nonlinear Klein-Gordon equation [6]

$$
u_{t t}-2.5 u_{x x}+u+1.5 u^{3}=0,
$$

with initial conditions

$$
u(x, 0)=B \tan (k x), \quad u_{t}(x, 0)=B c k \sec ^{2}(k x),
$$

where $B=\sqrt{\frac{\beta}{\gamma}}$ and $K=\sqrt{\frac{-\beta}{2\left(\alpha+c^{2}\right)}}$.

Equation (49) could be rewritten as

$$
u_{t t}-2.5 u_{x x}=-u-1.5 u^{3}
$$

Applying the Elzaki transform to both sides of equation (50)

$$
E\left[u_{t t}\right]-2.5 E\left[u_{x x}\right]=-E[u]-1.5 E\left[u^{3}\right]
$$




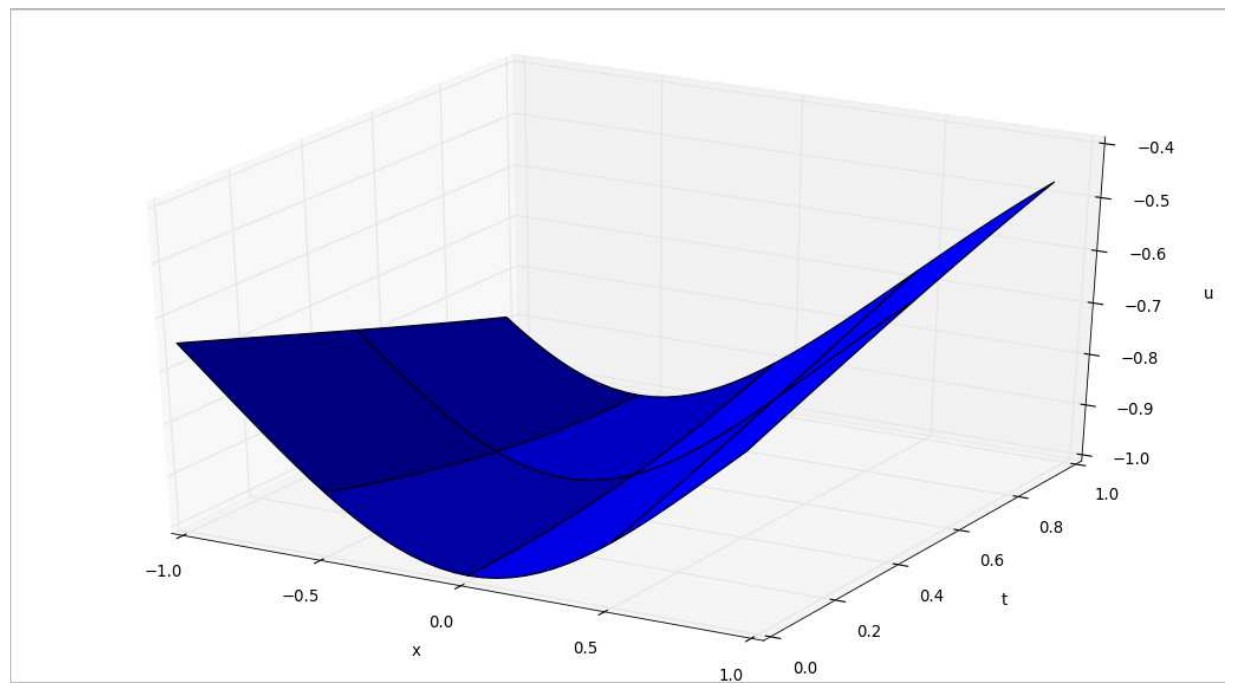

Figure 3: The solution of the third Klein-Gordon equation by ETM in equation (37)

where

$$
\begin{aligned}
& E\left[u_{t t}\right]=\frac{U(x, v)}{v^{2}}-u(x, 0)-v u_{t}(x, 0), \\
& E\left[u_{x x}\right]=\frac{d^{2}}{d x^{2}}[U(x, v)]=\frac{d^{2}}{d x^{2}} E[u],
\end{aligned}
$$

equation (51) becomes

$$
\frac{U(x, v)}{v^{2}}-u(x, 0)-v u_{t}(x, 0)-2.5 \frac{d^{2}}{d x^{2}} E[u]=-E[u]-1.5 E\left[u^{3}\right] .
$$

Applying the given initial conditions to equation (52) and simplifying, we obtain

$$
\begin{aligned}
U(x, v)= & v^{2} B \tan (k x)+v^{3} B c k \sec ^{2}(k x)-v^{2} E[u] \\
& +2.5 v^{2} \frac{d^{2}}{d x^{2}} E[u]-1.5 v^{2} E\left[u^{3}\right]
\end{aligned}
$$

Applying the inverse Elzaki transform to both side of equation (53) and simplifying, we have

$$
\begin{aligned}
u(x, t)= & B \tan (k x)+t B c k \sec ^{2}(k x)+ \\
& E^{-1}\left\{-v^{2} E\left[u_{n}\right]+2.5 v^{2} \frac{d^{2}}{d x^{2}} E\left[u_{n}\right]-1.5 v^{2} E\left[A_{n}\right]\right\} .
\end{aligned}
$$


From equation (54), let

$$
u_{0}=B \tan (k x)+t B c k \sec ^{2}(k x) .
$$

Now the recursive relation is given as

$$
u_{n+1}=E^{-1}\left\{-v^{2} E\left[u_{n}\right]+2.5 v^{2} \frac{d^{2}}{d x^{2}} E\left[u_{n}\right]-1.5 v^{2} E\left[A_{n}\right]\right\} .
$$

$A_{n}$ is the Adomian polynomial to decompose the nonlinear terms by using the relation

$$
A_{n}=\frac{1}{n !} \frac{d^{n}}{d \lambda^{n}} f\left[\sum_{i=0}^{\infty} \lambda^{i} u_{i}\right]_{\lambda=0}
$$

Let the nonlinear term be represented by

$$
f(u)=u^{3} .
$$

By using equation (58) in equation (57), we obtain

$$
A_{0}=u_{0}^{3}, \quad A_{1}=3 u_{1} u_{0}^{2}, \quad A_{2}=3 u_{0} u_{1}^{2}+3 u_{2} u_{0}^{2}, \quad \cdots .
$$

From equation (56), when $n=0$, we have

$$
u_{1}=E^{-1}\left\{-v^{2} E\left[u_{0}\right]+2.5 v^{2} \frac{d^{2}}{d x^{2}} E\left[u_{0}\right]-1.5 v^{2} E\left[A_{0}\right]\right\} .
$$

By simplifying equation (59) we obtain

$$
\begin{aligned}
& u_{1}=\frac{-B c K t^{3}}{12} \times \\
& {\left[1+4.5 B^{2}-20 K^{2}+\cos (2 K x)-4.5 B^{2} \cos (2 K x)+10 K^{2} \cos (2 K x)\right] \times} \\
& \sec ^{4}(K x)-\frac{B t^{2}}{4}\left[1+1.5 B^{2}-10 K^{2}+\cos (2 K x)-1.5 B^{2} \cos (2 K x)\right] \times \\
& \sec ^{2}(K x) \tan (K x)-\frac{1.5 B^{3} c^{3} K^{3} t^{5}}{20} \sec ^{6}(K x)- \\
& \frac{1.5 B^{3} c^{2} K^{2} t^{4}}{4} \sec ^{4}(K x) \tan (K x) .
\end{aligned}
$$

The approximate series solution is

$$
u(x, t)=u_{0}+u_{1}+\cdots .
$$

This series form can be expressed in a closed form as

$$
u(x, t)=B \tan (K(x+c t)) .
$$




\section{Conclusion}

The approximate analytical solutions of several Klein-Gordon equations have been obtained using the combination of the Elzaki transform method and Adomian polynomials which was meant for linearizing the nonlinear terms. The solutions obtained agree with the solutions obtained by Reduced differential transform method and radial basis functions as provided in the references. The three dimensional graphs were also plotted to demonstrate the solutions of Klein-Gordon equations considered. Moreover, the problems considered showed that the Elzaki transform method and Adomian polynomials can be very powerful tools in solving Klein-Gordon equations. This method is a promising method for solving other nonlinear partial differential equations.

Furthermore, the Elzaki transform just like other linear integral transform such as Laplace and Sumudu transform, which combined with the Adomian polynomials are useful for solving nonlinear differential equations. These linear integral transforms provide results in form of series which avoids discretization error. However, the transformation definitions of these methods differ from one to another.

\section{References}

[1] M.J. Ablowitz, M.A. Ablowitz, and P.A. Clarkson, Solitons, Nonlinear Evolution Equations and Inverse Scattering, Volume 149, Cambridge University Press, Cambridge (1991).

[2] K.C. Basak, P.C. Ray, and R.K. Bera, Solution of nonlinear Klein-Gordon equation with a quadratic nonlinear term by Adomian decomposition method, Communications in Nonlinear Science and Numerical Simulation, 14, No 3 (2009), 718-723.

[3] J. Biazar and H. Ghazvini, He's variational iteration method for solving hyperbolic differential equations, International Journal of Nonlinear Sciences and Numerical Simulation, 8, No 3 (2007), 311-314.

[4] N. Bildik and A. Konuralp, The use of variational iteration method, differential transform method and Adomian decomposition method for solving different types of nonlinear partial differential equations, International Journal of Nonlinear Sciences and Numerical Simulation, 7, No 1 (2006), 65-70.

[5] L. Debnath, Nonlinear Partial Differential Equations for Scientists and Engineers, Springer Science \& Business Media (2011).

[6] M. Dehghan and A. Shokri, Numerical solution of the nonlinear Klein-Gordon equation using radial basis functions, Journal of Computational and Applied Mathematics, 230, No 2 (2009), 400-410.

[7] O.E. Ige, M. Heilio, R.A. Oderinu and T.M. Elzaki, Adomian polynomial and Elzaki transform method of solving third order Korteweg-De Vries equations, Global Journal of Pure and Applied Mathematics, 15, No 3 (2019), 261-277. 
[8] O.E. Ige, R.A. Oderinu and T.M. Elzaki, Adomian polynomial and Elzaki transform method of solving fifth order Korteweg-De Vries equation, Caspian Journal of Mathematical Sciences, (2018), In press.

[9] P.G. Drazin and R.S. Johnson, Solitons: An Introduction, Volume 2, Cambridge University Press, Cambridge (1989).

[10] A. Ebaid, Exact solutions for the generalized Klein-Gordon equation via a transformation and exp-function method and comparison with Adomian's method, Journal of Computational and Applied Mathematics, 223, No 1 (2009), 278-290.

[11] S.M. El-Sayed, The decomposition method for studying the Klein-Gordon equation, Chaos, Solitons \& Fractals, 18, No 5 (2003), 1025-1030.

[12] T.M. Elzaki, Application of new transform "Elzaki transform" to partial differential equations, Global Journal of Pure and Applied Mathematics, 7, No 1 (2011), 65-70.

[13] T.M. Elzaki, The new integral transform "Elzaki transform", Global Journal of Pure and Applied Mathematics, 7, No 1 (2011), 57-64.

[14] T.M. Elzaki, On the connections between Laplace and Elzaki transforms, Advances in Theoretical and Applied Mathematics, 6, No 1 (2011), 1-11.

[15] T.M. Elzaki, On the Elzaki transform and ordinary differential equation with variable coefficients, Advances in Theoretical and Applied Mathematics, 6, No 1 (2011), 13-18.

[16] T.M. Elzaki, A solution for nonlinear systems of differential equations using a mixture of Elzaki transform and differential transform method, In International Mathematical Forum, 7 (2012), 625-630.

[17] T.M. Elzaki and S.A. Alkhateeb, Modification of Sumudu transform "Elzaki transform" and Adomian decomposition method, Applied Mathematical Sciences, 9, No 13 (2015), 603-611.

[18] T.M. Elzaki and E.M. Hilal, Homotopy perturbation and Elzaki transform for solving nonlinear partial differential equations, Mathematical Theory and Modeling, 2, No 3 (2012), 33-42.

[19] Y. Keskin, S. Servi, and G. Oturanc, Reduced differential transform method for solving Klein Gordon equations, In: Proc. of the World Congress on Engineering, 1 (2011), page 2011.

[20] Z. Odibat and S. Momani, A reliable treatment of Homotopy perturbation method for Klein-Gordon equations, Physics Letters A, 365, No 5-6 (2007), 351-357.

[21] S.S. Ray, An application of the modified decomposition method for the solution of the coupled Klein-Gordon-Schr odinger equation, Communications in Nonlinear Science and Numerical Simulation, 13, No 7 (2008), 1311-1317. 
[22] M. Suleman and Q. Wu, Transformed Homotopy Perturbation Method for Approximate Analytical Solution of Klein-Gordon and Sine-Gordon Equation (2015).

[23] Q. Sun, Solving the Klein-Gordon equation by means of the Homotopy analysis method, Applied Mathematics and Computation, 169, No 1 (2005), 355-365.

[24] Q. Wang and D. Cheng, Numerical solution of damped nonlinear Klein-Gordon equations using variational method and finite element approach, Applied Mathematics and Computation, 162, No 1 (2005), 381-401.

[25] A. Wazwaz, Partial Differential Equations, CRC Press (2002).

[26] E. Yusuf uglu, The variational iteration method for studying the Klein-Gordon equation, Applied Mathematics Letters, 21, No 7 (2008), 669-674.

[27] Y. Zhu, Q. Chang, and S. Wu, A new algorithm for calculating Adomian polynomials, Appl. Math. Comput., 169 (2005), 402-416.

[28] D. Ziane and M.H. Cherif, Resolution of nonlinear partial differential equations by Elzaki transform decomposition method, J. Appro. Theo. Appl. Math, 5 (2015), 17-30. 